\title{
The Uses of Carbon Nanotubes Mixed Matrix Membranes (MMM) for Biogas Purification
}

\author{
T.D. Kusworo ${ }^{1}$, A.F. Ismail ${ }^{2}$, Budiyono ${ }^{1,3}$, I.N. Widiasa ${ }^{1}$, S. Johari ${ }^{3}$, and Sunarso ${ }^{3}$ \\ ${ }^{1}$ Department of Chemical Engineering, \\ University of Diponegoro, Jl. Prof. Sudharto SH No. 1, Semarang, Indonesia \\ ${ }^{2}$ Advanced Membrane Research Technology Center, \\ Faculty of Chemical and Natural Resources Engineering, \\ Universiti Teknologi Malaysia, 81310 UTM, Skudai, Johor Bahru, Malaysia \\ ${ }^{3}$ Department of Animal Agriculture, \\ University of Diponegoro, Jl. Prof. Sudharto SH No. 1, Semarang, Indonesia
}

\begin{abstract}
A new type of mixed matrix membrane consisting of polyethersulfone (PES) and carbon nanotubes (CNTs) is prepared for biogas purification application. PES mixed matrix membrane with and without modification of carbon nanotubes were prepared by a dry/wet phase inversion technique using a pneumatically membrane casting machine system. The modified carbon nanotubes were prepared by treating the carbon nanotubes with chemical modification using acid treatment to allow PES chains to be grafted on carbon nanotubes surface. The results from the FESEM, DSC and FTIR analysis confirmed that chemical modification on carbon nanotubes surface had taken place. Meanwhile, the nanogaps in the interface of polymer and carbon nanotubes were appeared in the PES mixed matrix membrane with unmodified of carbon nanotubes. The modified carbon nanotubes mixed matrix membrane increases the mechanical properties and the permeability of all gases. For PES-modified carbon nanotubes mixed matrix membrane the maximum selectivity achieved for $\mathrm{CO}_{2} \mathrm{CH}_{4}$ is 23.54 .
\end{abstract}

Keyword: mixed matrix membrane, $\mathrm{CO}_{2}$ removal, carbon nanotubes, biogas purification

\section{INTRODUCTION}

One of the most important challenges that our world will face in the twenty-first century will be continuing to meet the ever increasing energy needs of its citizen. Along with the need to find a renewable long term energy source is the need to find a more environmental friendly one. One of the promising candidates as a power source solution for the future world energy problem is biomass. Biomass includes trees, crops, plants, agricultural and forest residue, wastes from food and beverage manufacturing effluents, animal waste, animal manure, sludge from waste water treatment plants and the organic fraction of domestic waste [1]. Common terminology to describe the biological origin of a product includes terms such as biofuel, bioenergy and biogas. There are three major categories of biochemical technologies developed for biomass or biogas production; ethanol fermentation, biodiesel production and anaerobic digestion. Anaerobic digestion (AD) is a natural process that utilizes methanogenic bacteria in an oxygen-limited environment to convert organic waste into biogas [2]. However, the use of biogas still has limitation due to lowquality of biogas containing substantial amounts of $\mathrm{CO}_{2}(20-$ $45 \mathrm{vol} \%)$ and $\mathrm{H}_{2} \mathrm{~S}(0-1 \mathrm{vol} \%)$ in addition to main component $\mathrm{CH}_{4}(55-80 \mathrm{vol} \%$

The utilization of biogas as an efficient energy source depends strongly on its methane concentration. Therefore, biogas purified and enriched in methane can be used for household applications, automobile fuel (liquefied) or electricity generation. There is a great need to make biogas transportable. This can be done by compressing the gas in cylinders, what is possible only after removing $\mathrm{CO}_{2}, \mathrm{H}_{2} \mathrm{~S}$ and water vapour. There is a lot of potential if biogas could be made viable as a transport vehicle fuel like CNG, by compressing and filling the cylinders in it after scrubbing and drying. Enrichment of methane in biogas to have fuel of higher calorific value can be achieved by removal of carbon dioxide. Elimination of carbon dioxide from the flue gas helps to increase its calorific value as well as to eliminate the greenhouse gas. The carbon dioxide thus generated can be utilised as an effective refrigerant. Current technologies to purify off-gas and increase its caloric value have been primarily limited to physicochemical methods such as chemical separation, membrane separation, cryogenic separation as well as adsorption.

Currently, membrane separation of gases has emerged into an important unit operations technique offering specific advantages over more conventional separation procedures such as absorption, distillation, scrubbing and amine treatment [3]. Membrane separation of gases technology offer a number of significant advantages such as membrane devices are almost always compact and modular, membrane system can be operated in mild conditions with simple equipment and membrane systems are energy efficient owing to a large reduction in power electricity and fuel consumption. Hybrid membranes composed organicinorganic has attracted attention as the future membrane material. Hybrid membranes or mixed matrix membrane concept combines the advantages of high separation capabilities of the molecular sieves and the desirable mechanical properties and economical processing capabilities of polymers [3-5]. Many studies have reported that the separation performance of mixed matrix membrane could be improved by integrating porous or nonporous inorganic filler such as zeolite, silica, carbon molecular sieve and activated carbon. Currently, significant efforts have been devoted in fabrication of mixed matrix membrane by use carbon nanotubes as great potential filler [6]. There are two basic types of CNT: single-wall carbon nanotubes (SWNT) and multiwall carbon nanotubes (MWNT) [7]. In this respect more attention of researchers is devoted towards 
for fabrication of the mixed matrix membranes by dispersing either single walled (SWNT) or multi walled (MWNT) carbon nanotubes into various polymer matrices. The properties of polymer nanocomposites containing carbon nanotubes depend on several factors in addition to the polymer: synthetic process used to produce nanotubes; nanotube purification process (if any); amount and type of impurities in the nanotubes; diameter, length, and aspect ratio of the nanotubes objects in the composite (isolated, ropes, and/or bundles); nanotubes orientation in the polymer matrix. Kim et al [8] studied the effect of incorporating of CNTs on polyimidesiloxane matrix on gas separation performance. They observed that the addition of small CNTs to the copolymer matrix will be reducing the permeability helium and hindering the diffusion of nitrogen due to the impermeable properties of CNTs.

At present, different approaches are reported to improve the dispersion of CNTs in solvents or polymers in order to fabricate the composite mixed matrix nanotubes membrane. Recent reports on the chemical compatibility and dissolution properties of CNTs have promoted a great deal of interests in developing modification and functionalization of their surface. The chemical functionalization of multiwall CNT can be done by using novel silane coupling agents which helps to covalently link polymers. Besides that, treatment by carboxylic acid groups also can be used in this modification but for the strong acid used it can defects on CNTs sidewalls and decrease the aspect ratio of CNTs and other possible surface modification techniques included plasma, thermal and laser ablation [9].

In the present study, the feasibility of mixed matrix membrane films using carbon nanotubes particles as selective inorganic fillers for biogas purification was investigated. The carbon nanotubes were functionalized using acid treatment. The aim is to get a CNTs linked with the coupling agent having a functional group such as a double bond which can be utilized further for copolymerization. Hence, this reaction product (CNTs silanization) can be chemically explored for improving their compatibility with polymer matrix.

\section{EXPERIMENTAL}

\section{A. Material selection}

Polyethersulfone was supplied by Solvay Advanced Material (USA). The polymers were dried in a vacuum oven at $120{ }^{\circ} \mathrm{C}$ overnight before dope preparation; N-methylpyrrolidinone (NMP) from Merck was used as the solvent due to its low toxicity. The Single wall nanotubes (SWNT) were produced using Thermal Catalytic Chemical Vapour Deposition (CCVD). The CCVD system is simple and includes a cost effective fixed bed flow reactor. The particle size of CNTs was about $25.76 \mathrm{~nm}$.

\section{B. Fabrication of asymmetric Polyether-sulfone-carbon} nanotubes mixed matrix membrane

In this study, the polymer solution consists of $25 \mathrm{wt} \%$ polymer, $75 \%$ NMP and $0.5-2$ wt $\%$ nanotubes in the total solid. The homogeneous polyethersulfone was prepared according to the following procedure; the unmodified or modified carbon nanotubes were dispersed into the solvent and stirred for 24 hours followed by the addition of a desired amount of polyethersulfone. The solution was agitated with a stirrer at least 24 hours to ensure complete dissolution of the polymer. This solution was further agitated by stirring at high speed for at least 2 days. Before casting, the homogeneously prepared solution was degassed under vacuums for 3 hours.

\section{Chemical modification method of carbon nanotubes surface}

The acid that be used in the study was sulphuric acid because it has many advantages. Refer to preparation of carbon nanotubes; both closed-ended and open-ended multi walled carbon nanotubes were purchased from carbon solutions. The raw carbon nanotubes materials were treated with sulphuric acid to purify CNTs from impurities before it mixed with the solvents and the polymer. Purification of CNTs is very important step before prepare the dope solution. As an example, the purification part for CNTs treatment with 1 molar sulphuric acid follows these steps. First, 1 molar sulphuric acid is prepared. About $5.56 \mathrm{~mL}$ of pure sulphuric acid $(18 \mathrm{M})$ is diluted with distilled water in $100 \mathrm{~mL}$ conical flask. Then, 0.25 to $0.30 \mathrm{~g}$ carbon nanotubes is mixed with the acid and it is stirred at least for four hours with temperature of $60^{\circ} \mathrm{C}$. After that, it must be dried in the oven with temperature of $60^{\circ} \mathrm{C}$ for a day. Then, it is ready to prepare the dope solution.

\section{POST-TREATMENT PROCEDURE}

The membrane sheets were coated with highly permeable elastomeric silicone polymer (Sylgard 184 Dow Corning). The membrane coating was done after the uncoated membranes were tested. The intention of coating is to fill any surface pinholes or defects on membrane surface. Membranes were submerged in the $3 \% \mathrm{w} / \mathrm{w}$ solution of silicone in n-hexane for 24 hours and subsequently placed in oven for 2 days at $60{ }^{\circ} \mathrm{C}$ to allow curing before permeation testing.

\section{E. Module fabrication and biogas permeation experiment}

The biogas permeation test involved the use of gas permeation cell in which the membrane was placed on a sintered metal plate and pressurized at the feed side. Gas permeation rates were measured by a constant pressure system using a soap bubble flow meter. The permeation test was used gas $\mathrm{CO}_{2}$ and $\mathrm{CH}_{4}$ as a model for biogas product. Figure 1 illustrates the gas permeation cell set up. The cross-membrane pressure difference was maintained 1 bar.

Pressure normalized gas permeation flux or permeance for gas $I,(P / l)_{I}$, in (GPU), can be calculated as follows:

$$
\left(\frac{P}{l}\right)_{i}=\frac{Q_{i}}{A \Delta p}
$$

where $Q_{i}$ is the volumetric flow rate of gas $i, \Delta p$ is pressure difference across membrane $(\mathrm{cmHg}), A$ is membrane affective surface area $\left(\mathrm{cm}^{2}\right)$ and $l$ is membrane skin thickness (cm). The ideal separation factor $\alpha_{i / j}$ can be calculated by using equation below:

$$
\alpha_{i / j}=\frac{(P / l)_{i}}{(P / l)_{j}}
$$




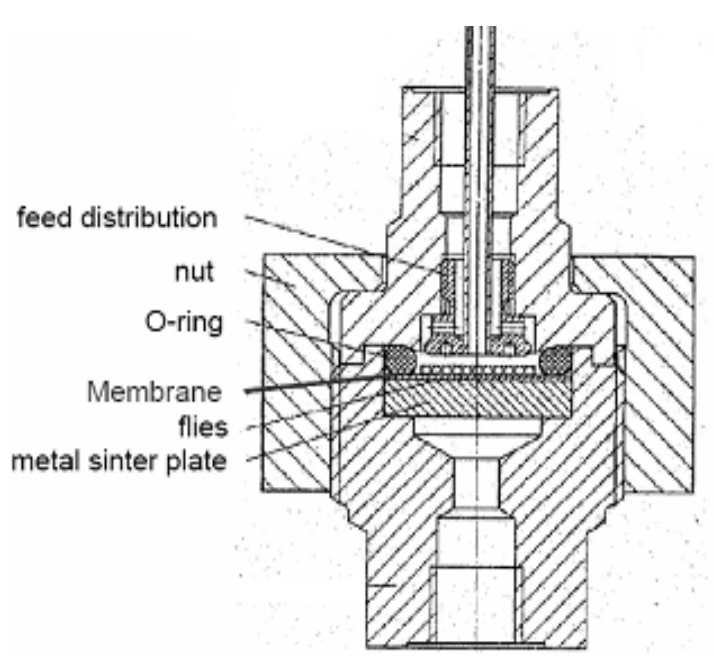

Fig. 1 Gas permeation test cell

\section{RESULTS AND DISCUSSION}

A. Morphology of asymmetric polyether-sulfone-nanotubes mixed matrix membranes

Figure 2 shows the field emission scanning electron microscopy (FESEM) micrographs of carbon nanotubes synthesize in Catalytic Chemical Vapour Deposition (CCVD) system using $\mathrm{SiNiAl}_{2} \mathrm{O}_{3}$ catalyst.

In Figure 2, the growth of CNTs can be clearly observed. The entangled and sinuous nanotubes are grown from the catalyst particles. The average diameter of the diameter of the as grown CNTs on the catalyst is $40 \mathrm{~nm}$. The CNTs can be seen as bundles of tubes with fluffy and spongy texture. The aggregation of CNTs in fabrication of polymer-nanotubes composites membranes can also be affected by the length and aspect ratio of CNTs [10]. Obviously, the as-grown nanotubes bundles are pure without purification, as no amorphous carbon and carbonaceous particles deposited on the catalyst. However, the impurities of metallic catalyst can be clearly showed on the surface of the ropes. Moreover, the gas flow through the mixed matrix membrane produced from CNTs without purification can be blocked with the metallic impurities.

The FESEM micrograph of CNTs after acid treatment is presented in Figure 3. From Figure 3 can be obtained that a clear distribution of bunches of CNTs is observed after chemical modification using acid treatment. As shown in Figure 3, the CNTs modified, the longer structure becomes shorter. Surface treatment of carbon nanotubes with sulphuric acid agent was carried out to produce silanol groups through hydrolysis reaction. Generally, the hydrolysis reaction occurs in the present of water molecules. Silanol groups formed as a product of a hydrolysis reaction react with hydroxyl groups found on nanotubes surfaces to form siloxane bonds (-Si-O-carbon nanotubes) through a condensation reaction.

In order to further investigate the effect of silanization of CNTs on the dispersion of CNTs in mixed matrix membrane, careful FESEM inspections were carried out. The FESEM micrographs of the cross-sectional and the surface of the PES-unmodified nanotubes and PESmodified nanotubes mixed matrix membranes are shown in Fig. 4-5.

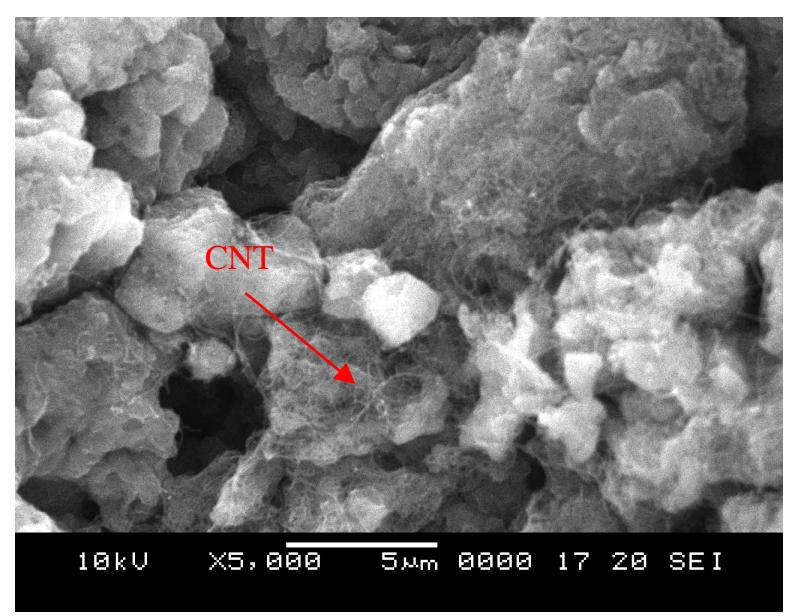

Fig. 2 FESEM image of unmodified CNTs

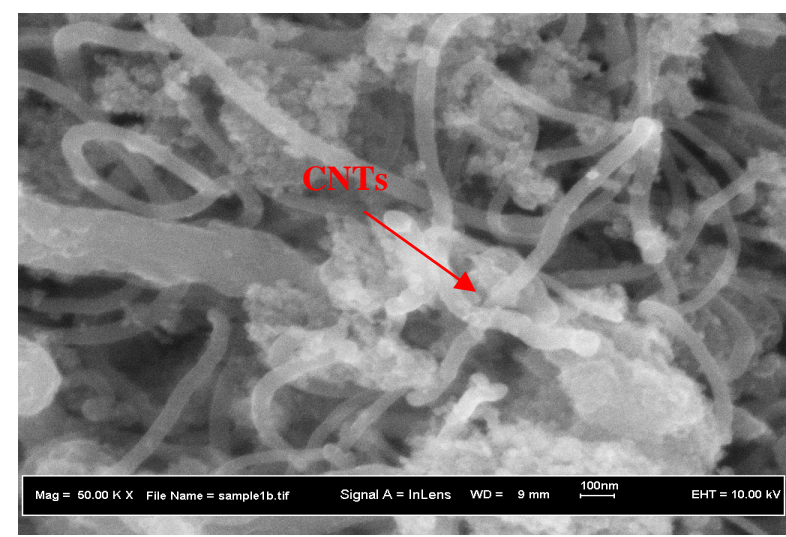

Fig. 3 FESEM image of CNTs after chemical modification using 2.5M sulphuric acid

As shown in Figures 4-5, structure of nanotubes composite membranes showing finger like structure which consisted of a dense skin layer supported by a spongy porous substructure. The unmodified carbon nanotubes particle seemed to good adhere with polymer matrix as shown in Figure 4. The smooth surface of the unmodified nanotubes-PI/PES mixed matrix membrane might be due to the shape of the carbon nanotubes particles appeared to be oblong, therefore no sharp edges that could easily perforated the active surface skin. However, in the case of unmodified carbon nanotubes, the unselective voids appeared indicated that the carbon nanotubes did not completely adhered on the surface of polymer matrix. The small surface ruptures and aggregation are also occurred on the mixed matrix membrane with unmodified carbon nanotubes as shown in Figure $4 b$.

The cross-section and surface of PES-modified CNTs using $2 \mathrm{wt} \%$ of CNTs are shown in Figure 5(a)-(b). The porous substrate of the fabricated membrane and the active skin layer can be seen in Figure 5. Cross-section of porous substrate region for PES modified CNT clearly show the compatibility between modified CNT loading with PES. Moreover, from the cross section can be seen that CNTs clearly act as the filler to in the PES polymer host. Due to the chemical and physical properties of amino silane could be able to enhance the bond strength which provides a stable and strong bond between the modified CNT with PES matrix and finally reduced the presence of voids surrounded on CNT surface. Therefore, the good compatibility between 
polymer matrix and carbon nanotubes would finally lead to diminish gas penetrants via unselective voids of carbon nanotubes and simultaneously high gas separation performance of membrane is able to be achieved. Figure 5 had also revealed the presence of some CNT under the skin layer due to the cutting in nitrogen. The smooth surface of carbon nanotubes might also induce to enhance the adhesion between the nanotubes and the host of polymer.

\section{B. Differential Scanning Calorimetry Analysis}

The Differential Scanning Calorimetry has been done to indicate whether the CNT-PES blends were indeed miscible by observing the glass transition temperatures of the blend composition. The $T_{g}$ for each membranes was determined from the heat flow versus temperature curve using an onset method. The influence of acid treatment modification on the glass transition temperature of PES-carbon nanotubes mixed matrix membranes are tabulated in Table 1. As shown in Table 1, the glass transition value, the acid treatmentfunctionalized CNT have increased the glass transition temperature $T_{g}$. This can be seen in the effect of $T_{g}$ for CNT modification with $2.5 \mathrm{M}$ sulphuric acid treatment. The increasing of $T_{g}$ in acid treatment-functionalized CNTs MMM is because of the strong interaction between the PES matrix and acid treatment-functionalized membrane which reduce the matrix mobility which will express itself in a shift of $T_{g}$ [11] and beside that the diffusion of small molecules can be retarded under high temperature. It can also be seen that compared with neat PES polymer, the $T_{g}$ of the mixed matrix membranes increased about $9{ }^{\circ} \mathrm{C}$ with the incorporating only $2 \mathrm{wt} \%$ modified carbon nanotubes using $2.5 \mathrm{M}$ sulphuric acid treatment into the matrix. This phenomenon indicates that the mobility of polymer chains is reduced due to the restriction effect of carbon nanotubes. This result is in agreement with the previous study [12].
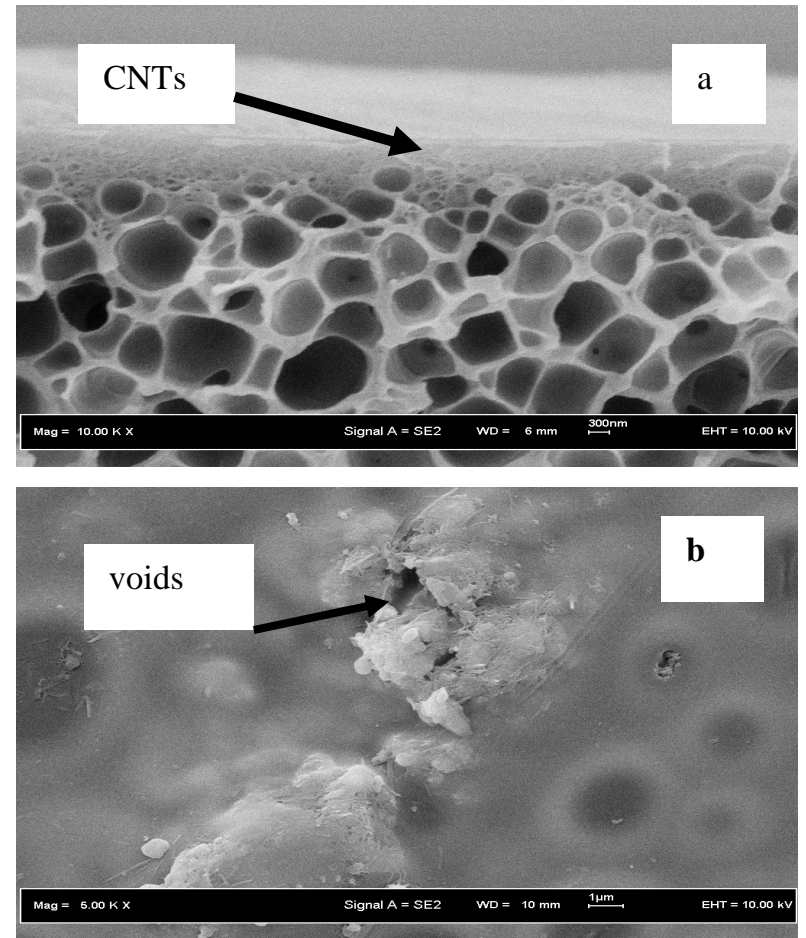

Fig. 4 SEM picture of asymmetric unmodified carbon nanotubespolyethersulfone mixed matrix membrane at the: (a) cross section and (b) outer surface image layer.
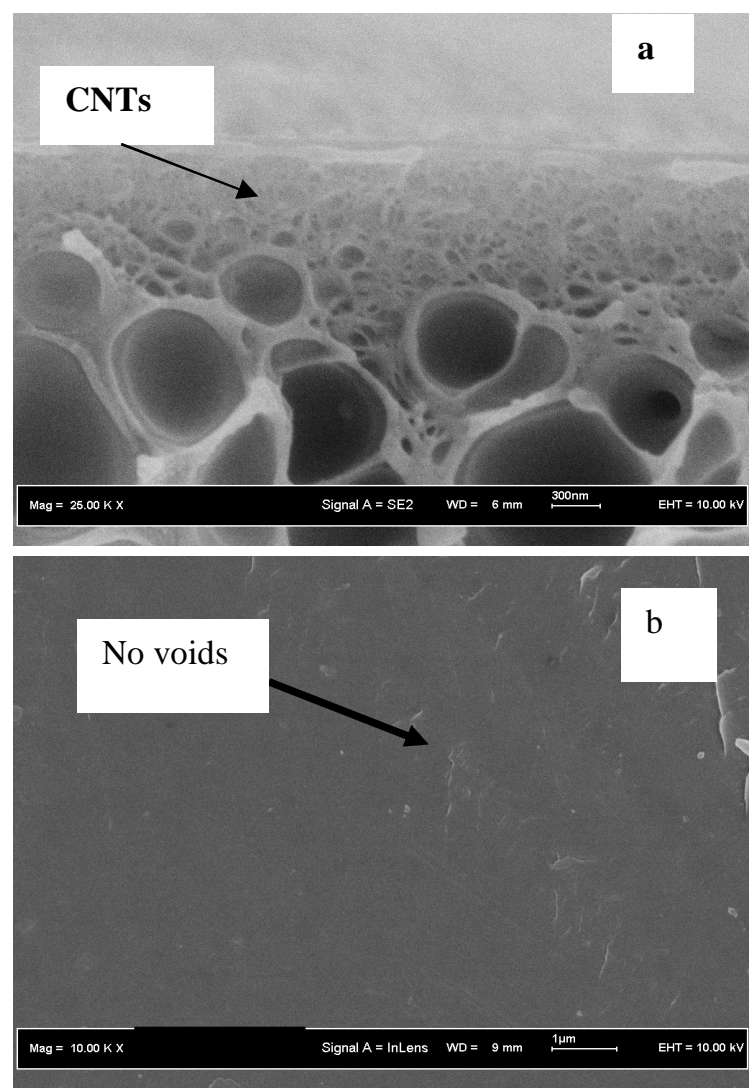

Fig. 5 SEM picture of asymmetric modified carbon nanotubespolyethersulfone mixed matrix membrane at the: (a) cross section and (b) outer surface image layer. TABLE 1

EFFECT OF SULPHURIC ACID TREATMENT OF CNTS ON THE GLASS TRANSITION TEMPERATURE OF PES-CNTS MIXED MATRIX MEMBRANE

\begin{tabular}{|l|c|}
\hline \multicolumn{1}{|c|}{ Membranes } & Tg ( $\left.{ }^{\mathbf{}} \mathbf{C}\right)$ \\
\hline PES & 218.8 \\
\hline Unmodified CNTs & 220.27 \\
\hline $\begin{array}{l}\text { Modified CNTs with 2.5 M } \\
\mathrm{H}_{2} \mathrm{SO}_{4}\end{array}$ \\
\hline
\end{tabular}

Based on Table 1, it can be concluded that the carbon nanotubes surface modification using sulphuric acid treatment could induced the adherence between polymer matrix and carbon nanotubes particles. Therefore, it can be concluded that modified carbon nanotubes using chemical modification included into PES matrix will be made the composite film stiffer and stronger due to strong interfacial interaction between the nanotubes and PES host matrix. Moreover, with addition the nanotubes the movement of polymer chain in polymer host matrix will be restricted and this phenomenon likes physical cross-linking.

\section{Biogas purification performance of PES-Carbon nanotubes mixed matrix membrane}

In the gas separation performance, the criteria which is important in determining whether the membrane are classified as good or not was determined by the ability of the membrane to give the high enhancement in gas separation performance than others membrane. Thus, this testing was carried out in order to study the membrane effectiveness due to the effect of chemical modification on carbon nanotubes surface. 
The membrane effectiveness in the gas separation performance was determined by the membrane permeability and selectivity for tested gas $\mathrm{CO}_{2} / \mathrm{CH}_{4}$. The gas permeation properties for carbon nanotubes mixed matrix membrane were measured by using variable pressure constant volume method. The permeability and selectivity for tested gases $\mathrm{CO}_{2} / \mathrm{CH}_{4}$ for PES-unmodified CNTs and PES-modified CNTs obtained were tabulated in Table 2.

Generally, the idea to put inorganic filler into organic polymer was enhanced gas permeability of polymer nanocomposites membranes was due to the disturbed polymer chain packing by the nanofillers [13]. Therefore, the well dispersed and good adherence of carbon nanotubes will be effectively increased the gas permeability due to more effectively insert between polymer chains of the matrix. The addition of $2 \mathrm{wt} \%$ of modified carbon nanotubes loading to polyethersulfone resulted in about $19.97 \%$ increases in permeability of $\mathrm{CO}_{2}$, while, the permeability of $\mathrm{CH}_{4}$ is increased up to $33.79 \%$, respectively. However, for the small gas molecules such as $\mathrm{CO}_{2}$ permeability slightly increased with the addition of carbon nanotubes in the polyethersulfone (PES) host matrix. The main pathways of gas transport through the mixed matrix membranes are through dense layer of PES matrix, highly selective carbon nanotubes and non-selective gaps or voids between the matrix and sieve particles. From FESEM data in Figure 3, the carbon nanotubes are well dispersed in polymer matrix and serve as channels to transport gas molecules. The permeability of $\mathrm{CH}_{4}$ exhibits the higher increasing of permeability compared to other gases. The results are consistent with the previous study done by previous studies [14-16]. They observed that the main factor affecting the increase of $\mathrm{CH}_{4}$ permeability with addition of carbon nanotubes into polymer host is due to the extremely fast diffusion of gas molecules adsorbed inside carbon nanotubes. They also reported that the SWNT membranes should be strongly selective for $\mathrm{CH}_{4}$ and would produce very large fluxes of $\mathrm{CH}_{4}$. Therefore, as presented in Table 2, the ideal selectivity for $\mathrm{CO}_{2} \mathrm{CH}_{4}$ of the PES-2 wt $\%$ carbon nanotubes was slightly increased due to preferential sorption of $\mathrm{CH}_{4}$ in the carbon nanotubes. Moreover, the permeability for unmodified carbon nanotubes for all gases also increases compared to neat PI/PES membrane. However, the $\mathrm{CO}_{2} / \mathrm{CH}_{4}$ selectivity was decreased for unmodified carbon nanotubes mixed matrix membrane. Therefore, the increase of permeability in the PESunmodified carbon nanotubes was not due to the gas transportation inside the nanotubes. As shown in Figure 4, the PES- unmodified carbon nanotubes are not well distributed in polymer matrix and the severe unselective voids between carbon nano-particles and polymer matrix was appeared. Hence, the PES chains could not fall onto the carbon nanotubes walls tightly and forming a narrow gaps surrounding the carbon nanotubes. Therefore, gas molecules can easily passed through the unselective voids or the gaps. The unselective voids would be functioned as pinholes that allow all gases molecules pass rapidly without any selectivity. Thus, the permeability of all gases is increased thus reducing the gas selectivity. As can be seen in Table 2, the sulphuric acid treated of modified carbon nanotubes filled PES polymer host mixed matrix membrane improved the $\mathrm{CO}_{2} / \mathrm{CH}_{4}$ selectivities.
TABLE 2

BIOGAS PERMEABILITY AND SELECTIVITY IN THE PES-

CARBON NANOTUBES MIXED MATRIX MEMBRANES

\begin{tabular}{|l|c|c|c|}
\hline \multirow{2}{*}{ Membrane } & \multicolumn{2}{|c|}{$\begin{array}{c}\text { Single gas permeance } \\
\text { (GPU) }\end{array}$} & Selectivity \\
\cline { 2 - 4 } & $\mathbf{C O}_{2}$ & $\mathbf{C H}_{4}$ & $\mathbf{C O}_{2} / \mathbf{C H}_{4}$ \\
\hline $\begin{array}{l}\text { PES neat } \\
\text { membrane }\end{array}$ & $33.45 \pm 1.15$ & $1.45 \pm 0.78$ & 23.07 \\
\hline $\begin{array}{l}\text { PES-unmodified } \\
\text { CNTs membrane }\end{array}$ & $37.79 \pm 0.78$ & $1.94 \pm 0.14$ & 19.48 \\
\hline $\begin{array}{l}\text { PES-modified } \\
\text { CNTs membrane }\end{array}$ & $54.03 \pm 0.78$ & $1.60 \pm 0.04$ & 33.76 \\
\hline
\end{tabular}

$*$ GPU $=1 \times 10^{-6} \mathrm{~cm}^{3}(\mathrm{STP}) / \mathrm{cm}^{2} \mathrm{~s} \mathrm{cmHg}$

\section{CONCLUSION}

In this study, the polyethersulfone (PES) with chemical modification of carbon nanotubes using sulphuric acid agent for the use of fabricating mixed matrix membrane for gas separation. Based on the experimental results and analysis, the following conclusions can be made.

* The FESEM for the cross-sectional and surface area images of mixed matrix membrane films indicated that the modified of carbon nanotubes dispersed well in the polymer matrix. The surface ruptures are not occurred on the carbon nanotubes mixed matrix membrane might be due to the shape of carbon nanotubes appeared to be oblong. The smooth surface of carbon nanotubes might also help to enhance the adherence between the nanotubes and the host polymer.

* The PES-modified carbon nanotubes membranes had increased the glass transition temperature, the permeability of all gases and the $\mathrm{CO}_{2} / \mathrm{CH}_{4}$ selectivity.

* The carbon nanotubes have been potentially as in organic filler for mixed matrix membrane for the future biogas separation membrane.

\section{REFERENCES}

[1]. G.V. Nallathambi, Anaerobic digestion of biomass for methane production: a review. Biomass and bioenergy, 13 (1997) 83-114

[2]. D.P., Chynoweth, J.M., Owens, and R. Legrand, Renewable methane from anaerobic digestion of biomass, Renewable Energy 22 (2001) 18.

[3]. P. Hacarlioglu, L. Toppare, L. Yilmaz, Polycarbonate-polypyrrole mixed matrix gas separation membranes. J. Membr. Sci., 225. (2003). 51-62.

[4]. C.M. Zimmerman, A. Singh., W.J. Koros, Tailoring mixed matrix composite membranes for gas separations. J. Membr. Sci., 137. (1997). 145-154.

[5]. T.D. Kusworo, A.F. Ismail, A. Mustafa and T. Matsuura, Dependence of Membrane Morphology and Performance on Preparation Conditions: The Shear Rate Effect in Membrane Casting, Separation and Purification Technology, 61. (2008) 249-257.

[6]. A.F. Ismail, T.D. Kusworo, A. Mustafa, Enhanced gas permeation performance of polyethersulfone mixed matrix hollow fiber membranes using novel Dynasylan Ameo silane agent, Journal of Membrane Science 319 (2008) 306-312.

[7]. Y.Lin, B. Zhou, K.A. Shiral Fernando, P. Liu, L.F. Allard, Y.-P. Sun, Polymeric carbon nanocomposites from carbon nanotubes functionalized with matrix polymer, Macromolecules, 36, (2003) 7199-7204

[8]. S. Kim, T.W. Pechar, E. Marand, Poly(imide siloxane) and carbon nanotube mixed matrix membranes for gas separation, Desalination, 192 (2006) 330-339.

[9]. P. C. Ma, J.-K. Kim, B. Z. Tang, Effects of silane functionalization on the properties of carbon nanotube/epoxy nanocomposites, Composites Science and Technology, 67, (2007) 2965-2972.

[10]. B.-K. Zhu, S.-H. Xie, Z.-K. Xu, Y.-Y. Xu, Preparation and properties of the polyimide/multi-walled carbon nanotubes 
(MWNTs) nanocomposites, Comp. Sci. Technol. 66 (2006) 548554.

[11]. Shen J., Huang W., Wu L., Hu Y., Ye M. (2007). The reinforcement role of different amino-functionalized multi-walled carbon nanotubes in epoxy nanocomposites. Comp. Sci. Tech. 67. 30413150

[12]. Y. Li, T.S. Chung, Z. Huang, S. Kulprathipanja, Dual-layer polyethersulfone (PES)/BTDA-TDI/MDI co-polyimide (P84) hollow fiber membranes with a submicron PES-zeolite beta mixed matrix dense-selective layer for gas separation, J. Membr. Sci., 227 (2006) 28-37.
[13]. D.Q. Vu, W.J. Koros, S.J. Miller, Mixed matrix membranes using carbon molecular sieves I. Preparation and experimental results, J. Membr. Sci., 211 (2003) 311-334.

[14]. H. Chen, D.S. Sholl, Prediction of selectivity and flux for CH4/H2 separations using single walled carbon nanotubes as membranes, J. Membr. Sci., 269 (2006) 152-160.

[15]. P.C. Ma, J-K. Kim, B.Z. Tang, Functionalization of carbon nanotubes using a silane coupling agent, Carbon, 44 (2006) 32323238 .

[16]. D.M. Delozier, K.A. Watson, J.G. Smith, Jr.T.C. Clancy, J.W. Connel, Investigation of aromatic/aliphatic polyimides as dispersants for single wall carbon nanotubes, Macromolecules, 39(2006)173 
